\title{
ABSTRACTS FROM CURRENT LITERATURE
}

\begin{abstract}
Determining the optimal strategy for reopening schools, the impact of test and trace interventions, and the risk of occurrence of a second COVID-19 epidemic wave in the UK: a modelling study
\end{abstract}

Jasmina Panovska-Griffiths, Cliff C Kerr, Robyn M Stuart, Dina Mistry, Daniel J Klein, Russell M Viner, Chris Bonell

Lancet Child Adolesc Health 2020;4:817-27.

Background: As lockdown measures to slow the spread of severe acute respiratory syndrome coronavirus 2 (SARS-CoV-2) infection begin to ease in the UK, it is important to assess the impact of any changes in policy, including school reopening and broader relaxation of physical distancing measures. We aimed to use an individual-based model to predict the impact of two possible strategies for reopening schools to all students in the UK from September, 2020, in combination with different assumptions about relaxation of physical distancing measures and the scale-up of testing.

Methods: In this modelling study, we used Covasim, a stochastic individual-based model for transmission of SARS-CoV-2, calibrated to the UK epidemic. The model describes individuals' contact networks stratified into household, school, workplace, and community layers, and uses demographic and epidemiological data from the UK. We simulated six different scenarios, representing the combination of two school reopening strategies (full time and a parttime rota system with $50 \%$ of students attending school on alternate weeks) and three testing scenarios $(68 \%$ contact tracing with no scale-up in testing, $68 \%$ contact tracing with sufficient testing to avoid a second COVID-19 wave, and $40 \%$ contact tracing with sufficient testing to avoid a second COVID-19 wave). We estimated the number of new infections, cases, and deaths, as well as the effective reproduction number $(R)$ under different strategies. In a sensitivity analysis to account for uncertainties within the stochastic simulation, we also simulated infectiousness of children and young adults aged younger than 20 years at $50 \%$ relative to older ages (20 years and older).
Findings: With increased levels of testing (between $59 \%$ and $87 \%$ of symptomatic people tested at some point during an active SARS-CoV-2 infection, depending on the scenario), and effective contact tracing and isolation, an epidemic rebound might be prevented. Assuming $68 \%$ of contacts could be traced, we estimate that $75 \%$ of individuals with symptomatic infection would need to be tested and positive cases isolated if schools return full-time in September, or $65 \%$ if a part-time rota system were used. If only $40 \%$ of contacts could be traced, these figures would increase to $87 \%$ and $75 \%$, respectively. However, without these levels of testing and contact tracing, reopening of schools together with gradual relaxing of the lockdown measures are likely to induce a second wave that would peak in December, 2020, if schools open full-time in September, and in February, 2021, if a part-time rota system were adopted. In either case, the second wave would result in $\mathrm{R}$ rising above 1 and a resulting second wave of infections $2 \cdot 0-2 \cdot 3$ times the size of the original COVID-19 wave. When infectiousness of children and young adults was varied from $100 \%$ to $50 \%$ of that of older ages, we still found that a comprehensive and effective test-trace-isolate strategy would be required to avoid a second COVID-19 wave.

Interpretation: To prevent a second COVID-19 wave, relaxation of physical distancing, including reopening of schools, in the UK must be accompanied by large-scale, population-wide testing of symptomatic individuals and effective tracing of their contacts, followed by isolation of diagnosed individuals.

\section{Neonatal management and outcomes during the COVID-19 pandemic: an observation cohort study}

Christine M Salvatore, Jin-Young Han, Karen P Acker, Priyanka Tiwari, Jenny Jin, Michael Brandler, Carla Cangemi, Laurie Gordon, Aimee Parow, Jennifer DiPace, Patricia DeLaMora

Lancet Child Adolesc Health 2020;4:721-27.

Background: The risk of vertical and perinatal transmission of severe acute respiratory syndrome coronavirus 2 (SARS-CoV-2, which causes COVID- 
19), the most appropriate management, and the neonate's risk of developing COVID-19 during the perinatal period are unknown. Therefore, we aimed to elucidate best practices regarding infection control in mother-newborn dyads, and identify potential risk factors associated with transmission.

Methods: In this observational cohort study, we identified all neonates born between March 22 and May 17, 2020, at three New York Presbyterian Hospitals in New York City (NY, USA) to mothers positive for SARS-CoV-2 at delivery. Mothers could practice skin-to-skin care and breastfeed in the delivery room, but had to wear a surgical mask when near their neonate and practice proper hand hygiene before skin-to-skin contact, breastfeeding, and routine care. Unless medically required, neonates were kept in a closed Giraffe isolette in the same room as their mothers, and were held by mothers for feeding after appropriate hand hygiene, breast cleansing, and placement of a surgical mask. Neonates were tested for SARS-CoV-2 by use of realtime PCR on nasopharyngeal swabs taken at $24 \mathrm{~h}$, 5-7 days, and 14 days of life, and were clinically evaluated by telemedicine at 1 month of age. We recorded demographics, neonatal, and maternal clinical presentation, as well as infection control practices in the hospital and at home.

Findings: Of 1481 deliveries, 116 (8\%) mothers tested positive for SARS-CoV-2; 120 neonates were identified. All neonates were tested at $24 \mathrm{~h}$ of life and none were positive for SARS-CoV-2. 82 (68\%) neonates completed follow-up at day $5-7$ of life. Of the 82 neonates, 68 (83\%) roomed in with the mothers. All mothers were allowed to breastfeed; at 5-7 days of life, 64 (78\%) were still breastfeeding. 79 (96\%) of 82 neonates had a repeat PCR at 5-7 days of life, which was negative in all; $72(88 \%)$ neonates were also tested at 14 days of life and none were positive. None of the neonates had symptoms of COVID-19.

Interpretation: Our data suggest that perinatal transmission of COVID-19 is unlikely to occur if correct hygiene precautions are undertaken, and that allowing neonates to room in with their mothers and direct breastfeeding are safe procedures when paired with effective parental education of infant protective strategies.
Intensive care admissions of children with paediatric inflammatory multisystem syndrome temporally associated with SARSCoV-2 (PIMS-TS) in the UK: a multicentre observational study

Patrick Davies, Claire Evans, Hari Krishnan Kanthimathinathan, Jon Lillie, Joseph Brierley, Gareth Waters, Mae Johnson, Benedict Griffiths, Pascale du Pré, Zoha Mohammad, Akash Deep, Stephen Playfor, Davinder Singh, David Inwald, Michelle Jardine, Oliver Ross, Nayan Shetty, Mark Worrall, Ruchi Sinha, Ashwani Koul, Elizabeth Whittaker, Harish Vyas, Barnaby R Scholefield, Padmanabhan Ramnarayan

\section{Lancet Child Adolesc Health 2020;4:669-77.}

Background: In April, 2020, clinicians in the UK observed a cluster of children with unexplained inflammation requiring admission to paediatric intensive care units (PICUs). We aimed to describe the clinical characteristics, course, management, and outcomes of patients admitted to PICUs with this condition, which is now known as paediatric inflammatory multisystem syndrome temporally associated with SARS-CoV-2 (PIMS-TS).

Methods: We did a multicentre observational study of children (aged $<18$ years), admitted to PICUs in the UK between April 1 and May 10, 2020, fulfilling the case definition of PIMS-TS published by the Royal College of Paediatrics and Child Health. We analysed routinely collected, de-identified data, including demographic details, presenting clinical features, underlying comorbidities, laboratory markers, echocardiographic findings, interventions, treatments, and outcomes; serology information was collected if available. PICU admission rates of PIMSTS were compared with historical trends of PICU admissions for four similar inflammatory conditions (Kawasaki disease, toxic shock syndrome, haemophagocytic lymphohistiocytosis, and macrophage activation syndrome).

Findings: 78 cases of PIMS-TS were reported by 21 of 23 PICUs in the UK. Historical data for similar inflammatory conditions showed a mean of one (95\% CI 0.85-1.22) admission per week, compared to an average of 14 admissions per week for PIMS-TS and a peak of 32 admissions per week during the study 
period. The median age of patients was 11 years (IQR $8-14$ ). Male patients (52 [67\%] of 78) and those from ethnic minority backgrounds (61 [78\%] of 78) were over-represented. Fever (78 [100\%] patients), shock (68 [87\%]), abdominal pain (48 [62\%]), vomiting (49 [63\%]), and diarrhoea (50 [64\%]) were common presenting features. Longitudinal data over the first 4 days of admission showed a serial reduction in Creactive protein (from a median of $264 \mathrm{mg} / \mathrm{L}$ on day 1 to $96 \mathrm{mg} / \mathrm{L}$ on day 4), D-dimer (4030 ig/L to 1659 ìg/L), and ferritin (1042 ig/L to $757 \mathrm{ig} / \mathrm{L})$, whereas the lymphocyte count increased to more than $1.0 \times$ $10^{9}$ cells per L by day 3 and troponin increased over the 4 days (from a median of $157 \mathrm{ng} / \mathrm{mL}$ to $358 \mathrm{ng} /$ $\mathrm{mL}) .36(46 \%)$ of 78 patients were invasively ventilated and 65 (83\%) needed vasoactive infusions; $57(73 \%)$ received steroids, $59(76 \%)$ received intravenous immunoglobulin, and $17(22 \%)$ received biologic therapies. 28 (36\%) had evidence of coronary artery abnormalities (18 aneurysms and ten echogenicity). Three children needed extracorporeal membrane oxygenation, and two children died.

Interpretation: During the study period, the rate of PICU admissions for PIMS-TS was at least 11-fold higher than historical trends for similar inflammatory conditions. Clinical presentations and treatments varied. Coronary artery aneurysms appear to be an important complication. Although immediate survival is high, the long-term outcomes of children with PIMS-TS are unknown.

\section{Emergence of Kawasaki disease related to SARS-CoV-2 infection in an epicentre of the French COVID-19 epidemic: a time-series analysis}

Naim Ouldali, Marie Pouletty, Patricia Mariani, Constance Beyler, Audrey Blachier, Stephane Bonacorsi, Kostas Danis, Maryline Chomton, Laure Maurice, Fleur Le Bourgeois, Marion Caseris, Jean Gaschignard, Julie Poline, Robert Cohen, Luigi Titomanlio, Albert Faye, Isabelle Melki, Ulrich Meinzer

Lancet Child Adolesc Health 2020;4:662-68.

Background: Kawasaki disease is an acute febrile systemic childhood vasculitis, which is suspected to be triggered by respiratory viral infections. We aimed to examine whether the ongoing COVID-19 epidemic, caused by severe acute respiratory syndrome coronavirus 2 (SARS-CoV-2), is associated with an increase in the incidence of Kawasaki disease.

Methods: We did a quasi-experimental interrupted time series analysis over the past 15 years in a tertiary paediatric centre in the Paris region, a French epicentre of the COVID-19 outbreak. The main outcome was the number of Kawasaki disease cases over time, estimated by quasi-Poisson regression. In the same centre, we recorded the number of hospital admissions from the emergency department (2005-2020) and the results of nasopharyngeal multiplex PCR to identify respiratory pathogens (2017-2020). These data were compared with daily hospital admissions due to confirmed COVID-19 in the same region, recorded by Public Health France.

Findings: Between Dec 1, 2005, and May 20, 2020, we included 230 patients with Kawasaki disease. The median number of Kawasaki disease hospitalisations estimated by the quasi-Poisson model was 1.2 per month (IQR 1·1-1·3). In April, 2020, we identified a rapid increase of Kawasaki disease that was related to SARS-CoV-2 (six cases per month; $497 \%$ increase [95\% CI 72-1082]; $p=0 \cdot 0011$ ), starting 2 weeks after the peak of the COVID-19 epidemic. SARS-CoV-2 was the only virus circulating intensely during this period, and was found in eight (80\%) of ten patients with Kawasaki disease since April 15 (SARS-CoV-2-positive PCR or serology). A second peak of hospital admissions due to Kawasaki disease was observed in December, 2009 (six cases per month; 365\% increase ([31-719]; $p=0.0053)$, concomitant with the influenza A H1N1 pandemic.

Interpretation: Our study further suggests that viral respiratory infections, including SAR-CoV-2, could be triggers for Kawasaki disease and indicates the potential timing of an increase in incidence of the disease in COVID-19 epidemics. Health-care providers should be prepared to manage an influx of patients with severe Kawasaki disease, particularly in countries where the peak of COVID-19 has recently been reached. 


\section{Epidemiological and Clinical Profile of Pediatric Inflammatory Multisystem Syndrome - Temporally Associated with SARS- CoV-2 (PIMS-TS) in Indian Children}

K Dhanalakshmi, Aishwarya Venkataraman, S Balasubramanian, Manoj Madhusudan, Sumanth Amperayani, Sulochana Putilibai, Kalaimaran Sadasivam, Bala Ramachandran and AV Ramanan

Indian Pediatr 2020;57:1010-14.

Background: We describe the demographic, clinical and labo-ratory findings along with the treatment and outcomes among children meeting the case definition of Pediatric Inflammatory Multisystem Syndrome - Temporally associated with SARS-CoV2 (PIMS-TS).

Methods: We analyzed the clinical and laboratory findings of children who presented with PIMS-TS during an 8-week period from May 4, 2020 to July 8, 2020.

Results: We report 19 children with a median age of 6 year (IQR: 13 months-16 years), who met the case definition of PIMS-TS. All of them presented with fever. Multi organ involvement (79\%), mucocutaneous involvement (74\%), cardiovascular symptoms (63\%) and gastrointestinal symptoms (42\%) were the other features. Elevated levels of C-reactive protein was found in all of them and the majority of them had evidence of coagulopathy; intensive care admissions were needed in $12(63 \%)$ and vasoactive medications were given to $6(31.5 \%)$ children. There were no deaths.

Conclusion: Children with PIMS-TS present with a wide range of signs and symptoms. Fewer children in this series had coronary artery abnormalities, and there was a low incidence of RT-PCR positivity with high presence of SARS-CoV-2 antibodies.
Multisystem Inflammatory Syndrome in Children With COVID-19 in Mumbai, India

Shreepal Jain, Supratim Sen, Srinivas Lakshmivenkateshiah, Prashant Bobhate, Sumitra Venkatesh, Soonu Udani, Laxmi Shobhavat, Parmanand Andankar, Tanuja Karande and Snehal Kulkarni

Indian Pediatr 2020;57:1015-19

Objective: We describe the presentation, treatment and outcome of children with multisystem inflammatory syndrome with COVID-19 (MIS-C) in Mumbai metropolitan area in India.

Methods: This is an observational study conducted at four tertiary hospitals in Mumbai. Parameters including demographics, symptomatology, laboratory markers, medications and outcome were obtained from patient hospital records and analyzed in patients treated for MIS-C (as per WHO criteria) from 1 May, 2020 to 15 July, 2020.

Results: 23 patients (11 males) with median (range) age of $7.2(0.8-14)$ years were included. COVID-19 RT-PCR or antibody was positive in $39.1 \%$ and $30.4 \%$, respectively; $34.8 \%$ had a positive contact. $65 \%$ patients presented in shock; these children had a higher age $(P=0.05)$, and significantly higher incidence of myocarditis with elevated troponin, NT pro BNP and left ventri-cular dysfunction, along with significant neutrophilia and lympho-penia, as compared to those without shock. Coronary artery dilation was seen in $26 \%$ patients overall. Steroids were used most commonly for treatment (96\%), usually along with intra-venous immunoglobulin (IVIg) (65\%). Outcome was good with only one death.

Conclusion: Initial data on MIS-C from India is presented. Further studies and longer surveillance of patients with MIS-C are required to improve our diagnostic, treatment and surveillance criteria. 